\title{
Carotid Embolectomy and Endarterectomy for Symptomatic Complete Occlusion of the Carotid Artery as a Rescue Therapy in Acute Ischemic Stroke
}

\author{
Sami Curtze ${ }^{a} \quad$ Jukka Putaala ${ }^{a}$ Marika Saarela $^{a}$ \\ Pirkka Vikatmaa $^{b} \quad$ Ilkka Kantonen $^{b}$ Turgut Tatlisumak ${ }^{a}$ \\ Departments of ${ }^{a}$ Neurology, and ${ }^{b}$ Vascular Surgery, Helsinki University Central \\ Hospital, Helsinki, Finland
}

\section{Key Words}

Internal carotid artery - Carotid endarterectomy - Acute stroke treatment . Embolectomy

\begin{abstract}
Emergency endarterectomy of an occluded internal carotid artery (ICA) has not been investigated as an option of rescue therapy for severe acute ischemic stroke in the era of intravenous (IV) thrombolysis treatment neither as a primary treatment nor after failed IV thrombolysis. Data from the pre-IV thrombolysis era are conflicting and therefore emergency endarterectomy has not been recommended. The number of patients reaching the emergency room within the IV thrombolysis time window has vastly grown due to advanced acute stroke treatment protocols. The efficacy of mechanical thrombectomy as a primary or add-on to IV thrombolysis therapy option is being actively investigated. We herein report 2 cases of acute ischemic stroke with computerized tomography (CT) angiography-documented occlusion of an ICA that were treated with emergency carotid endarterectomy and embolectomy to restore cerebral blood flow. Both cases presented with severe stroke symptoms and signs not responding to IV thrombolysis and showed severe CT-perfusion deficits mainly representing ischemic penumbra. Blood flow was surgically restored after $5 \mathrm{~h}$ of symptom onset. Both patients achieved a favorable outcome. We conclude that timely surgical approach of acute ICA occlusion after failed thrombolysis as a rescue therapy may be a viable option in well-selected patients.
\end{abstract}




\section{Introduction}

Acute ischemic stroke is currently treated with intravenous (IV) thrombolysis if the treatment can be given within the first $4.5 \mathrm{~h}$ of symptom onset [1]. Availability of thrombolysis has led to growing patient numbers reaching the emergency room within the IV thrombolysis time window due to advanced acute stroke treatment protocols and streamlined patient management. Patients not responding to IV thrombolysis, those unlikely to respond to IV thrombolysis, and few others undergo various rescue therapies, mostly mechanical thrombectomy, although their efficacy remains to be proven [2].

Surgical endarterectomy of a carotid artery stenosis or occlusion (CEA, carotid endarterectomy) has been investigated since the 1960s, but the role of emergency endarterectomy of total occlusions has been controversial and largely debated in the 1980s [3]. Few attempts have been made to recanalize an acute carotid artery occlusion within a time window of 48-72 h after symptom onset. Urgent CEA to prevent stroke recurrence and improve neurological outcome in transient ischemic attack and acute ischemic stroke patients has been recently investigated in nonthrombolyzed patients $[4,5]$, including 3 patients with occlusion of the artery [5]. In one study, 10 patients underwent CEA within $6 \mathrm{~h}$ from symptom onset. However, no significant difference emerged in outcome compared with those 18 treated with CEA after $6 \mathrm{~h}$ of symptom onset [4].

We present two cases of carotid artery occlusion and acute ischemic stroke where surgical approach was employed within a reasonable time window upon failure of IV thrombolysis.

\section{Case}

Case 1

A 71-year-old, right-handed white female suffering from hypothyroidism and hypertension had been investigated because of acute aphasia and right hand motor symptoms caused by an ischemic stroke 14 months earlier with a favorable recovery, scoring 1 on the modified Rankin Scale (mRS). Etiologic work-up revealed atherosclerotic left carotid artery stenosis of less than $50 \%$ according to NASCET (North American Symptomatic Carotid Endarterectomy Trial) criteria. She had a history of a documented episode of atrial fibrillation, but on the $48 \mathrm{~h}$ ambulatory ECG no fibrillations were detected. She refused long-term oral anticoagulation.

Fourteen months later she developed sudden aphasia and severe right-sided hemiparesis with onset at 09:30 AM. She presented at the emergency room at 10:53 AM scoring 19 points on the NIH Stroke Scale (NIHSS). She was unable to talk and had right-sided hemiplegia. A CT scan of the brain revealed no early infarct signs or dense vessels, nor any radiological contraindications against IV thrombolysis. She received standard IV alteplase treatment, starting at $11: 04 \mathrm{AM}(0.9 \mathrm{mg} / \mathrm{kg}$ body weight, $10 \%$ as a bolus and $90 \%$ as $1 \mathrm{~h}$ continuous infusion).

As she did not improve clinically soon after IV alteplase infusion, she underwent brain imaging for possible further interventions. Occlusion of the left common carotid artery (CCA) and internal carotid artery (ICA) was found on computed tomography angiography ( fig. 1a), but the left middle cerebral artery (MCA) and the top of the ICA demonstrated retrograde contrast medium filling via collaterals (fig. 1b). Perfusion CT revealed significantly increased time to drain (TTD) and significantly decreased cerebral blood flow (CBF) in the whole left MCA and anterior cerebral artery territories (fig. 1c, d). Cerebral blood volume (CBV) maps showed a small infarct core within the disturbed perfusion territory (fig. 1e) 
Thereafter, the patient was immediately transferred to the operating room where her symptoms remained unchanged (NIHSS score 19). She was intubated and surgery started at 01:00 PM.

The surgery of the left carotid artery was performed under general anesthesia with no shunting. The left carotid bifurcation was identified at about 01:30 PM. After application of unfractionized heparin $(7,500 \mathrm{IU})$ the CCA and the external carotid artery (ECA) were clamped before incision of the vessel. A fresh thrombus of about $2 \mathrm{~cm}$ in length and $1 \mathrm{~cm}$ in thickness was detached from the left carotid artery bifurcation. The ICA was not clamped before thrombectomy and confirmed retrograde blood flow from the ICA. The residual atherosclerotic ICA stenosis of about $50 \%$ was endarterectomized. Blood flow to the brain through the left ICA was opened $5 \mathrm{~h}$ after onset of the symptoms at 02:30 PM, a few seconds after reopening the ECA. The blood flow of the ICA was measured to be $320 \mathrm{ml} / \mathrm{min}$ at this point in time. After extubation, the patient was transferred to our stroke unit.

At post-operative day 1 , she was able to speak and to move all extremities reaching 5 points on the NIHSS scale. The left leg was slightly weak; she had mild aphasia, and dysarthria. Furthermore, she answered incorrectly to age (by 1 year) and current month (by 1 month) questions.

On post-operative day 2, majority of the acute symptoms had resolved and the patient was in a condition comparable to before the acute stroke. Cardiogenic embolism due to atrial fibrillation was considered to be the most likely etiology for ICA occlusion with concomitant underlying atherosclerotic changes in the vessel wall. She was discharged home at day 7.

MRI at day 7 showed leukoaraiosis with severity of grade 2 according to the Fazekas Scale and patchy infarctions in the left MCA territory (fig. 2a-c). These small infarcted areas were not bright on diffusion imaging and might partially represent findings from the episode 14 months before the current evaluation. MRA demonstrated normal flow in the treated ICA (fig. 2d).

A telephone interview with the patient and her husband at 1 month confirmed mRS 1 with mild residual symptoms in the right hand. The patient is currently on continuous oral anticoagulation medication.

Case 2

A 72-year-old white male suffering from ulcerous colitis and chronic atrial fibrillation had been investigated because of acute dysarthria, aphasia, and right hand weakness 3 years prior to current admission. He had suffered from an acute ischemic stroke from which he recovered to mRS 1 . Etiologic work-up had revealed $50-70 \%$ atherosclerotic stenosis in the asymptomatic right ICA. He had presented with chronic atrial fibrillation and has been on oral anticoagulation medication since then.

Three years later, following an interruption of anticoagulation for 3 days for a routine colonoscopy, he suffered from acute vertigo and left-sided hemiparesis at 03:45 AM. The acute symptoms resolved completely at 05:00 AM when he reached our hospital. However, at 08:14 AM he presented with fluctuating left-sided hemiparesis that resolved quickly and at 11:05 AM again with acute severe left-sided hemiparesis and neglect (NIHSS 9), which led to IV thrombolysis at 12:10 PM. A CT scan of the brain did not reveal contraindications for IV thrombolysis nor did it show early infarct signs or dense vessel signs. International normalized ratio at admission was 1.5.

Because of severe stroke symptoms he was evaluated for the possibility of further therapy. There was an occlusion of the right ICA (fig. 3a) but right MCA and the top of the ICA had retrograde filling by means of collaterals with contrast medium (fig. 3b). Perfusion CT showed significantly increased TTD and significantly decreased CBF in the whole right MCA territory (fig. 3 c, d). CBV map showed decreased values at the core of the hypoperfusion region suggesting a small infarct core within a large ischemic penumbra (fig. 3e).

The patient was transferred to the operating room where he was intubated and surgery started at 02:45 PM, without any preceding clinical improvement (NIHSS score was 10 immediately before the induction of anesthesia). The surgery was performed under general anesthesia with no shunting. The right carotid bifurcation was identified. Unfractionized heparin (7,500 IU) was administered before clamping of the right CCA and the ECA. A fresh thrombus was detached from the right ICA. The ICA 
was not clamped before thrombectomy and confirmed retrograde blood flow from the ICA. Routine endarterectomy with patch angioplasty was also performed to moderate carotid artery stenosis.

Blood flow to the brain through the right ICA was opened at 04:00 PM a few seconds after opening the ECA. The blood flow of the ICA was measured to be $120 \mathrm{ml} / \mathrm{min}$ at this point in time. Blood flow was restored about $12 \mathrm{~h}$ after the initial and $5 \mathrm{~h}$ after the reappearance of the symptoms. He was transferred directly to our stroke unit from the operation room.

At day 1 he was able to speak and to move his arms and legs, scoring 5 on the NIHSS. The left hand was slightly weak and the patient had mild left facial paresis, dysarthria, and sensory deficit.

Cardiogenic embolism due to atrial fibrillation and interrupted anticoagulation was considered to be the most likely etiology. At day 7, he scored 3 points on the NIHSS score (slight weakness of the left arm, mild facial paresis, and dysarthria; 1 point for each).

A repeated CT scan at $24 \mathrm{~h}$ and MRI at day 6 (fig. $4 \mathrm{a}-\mathrm{c}$ ) depicted a modest-sized right cortical acute infarct in the MCA territory precisely corresponding to the CBV deficit observed on preoperative perfusion-CT image (fig. 3d). MRA demonstrated normal flow in the treated ICA (fig. 4d). The patient is currently on oral anticoagulation medication.

A telephone interview with the patient at 1 month confirmed mRS 2 with mild symptoms in the right hand.

\section{Discussion}

Our patients were considered for rescue therapy by means of endovascular mechanical thrombectomy after IV thrombolysis for a major ischemic stroke. The evaluation revealed total occlusion and no suspicion for dissection of CCA in one and of the ICA in the other case. A large thrombus bears the risk of breaking up into small thrombi that might occlude intracranial vessels after a thrombectomy attempt with endovascular catheters. Therefore, we decided to remove the thrombus surgically with concomitant endarterectomy. As this procedure has not been performed in our institution as a rescue therapy for acute ischemic stroke before, there was some delay in starting the surgery. However, we were able to restore reperfusion within $5 \mathrm{~h}$ after onset of symptoms and the patients recovered better than expected. At the moment, an emergency endarterectomy is an option in selected patients in our institution. Criteria for the intervention include acute severe stroke in a patient having significantly reduced flow in the ipsilateral anterior circulation without evidence of intracranial thrombi, but total ipsilateral ICA occlusion presumably of cardiac embolic origin.

\section{Conclusion}

A fast surgical approach to acute ICA or CCA occlusion after failed thrombolysis might be a reasonable rescue therapy for carefully selected patients. 


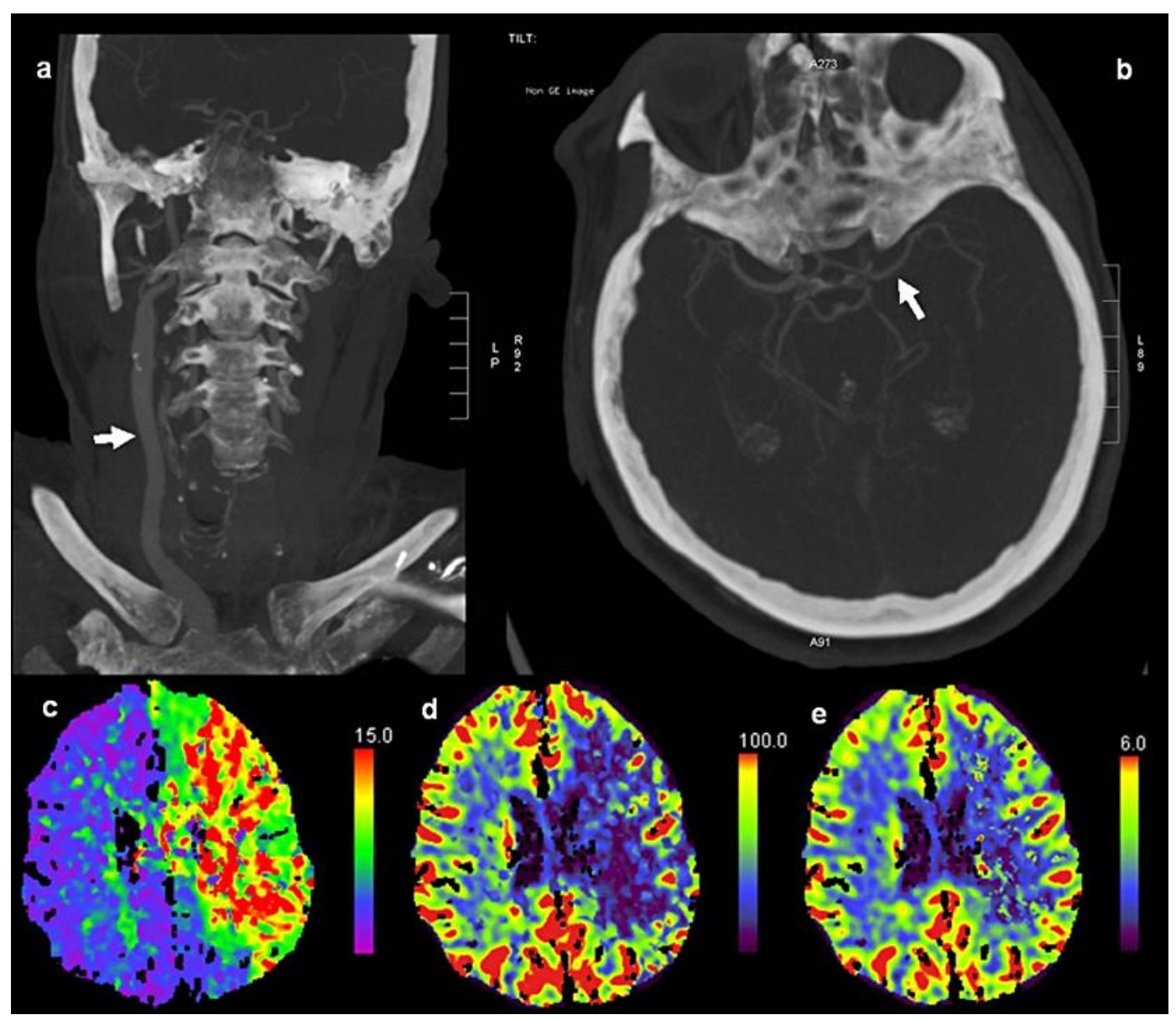

Fig. 1. Eighty minutes after onset of right-sided hemiparesis and aphasia (patient 1). CT angiogram shows normal contrast enhancement of the right carotid artery (arrow) but missing enhancement on the left side indicating total occlusion of the left carotid artery (a). Large intracranial vessels show contrast enhancement indicating collateral flow (arrow) in the left MCA (b). TTD (c), CBF (d), and CBV (e) maps show severe perfusion defect of the left hemisphere. 


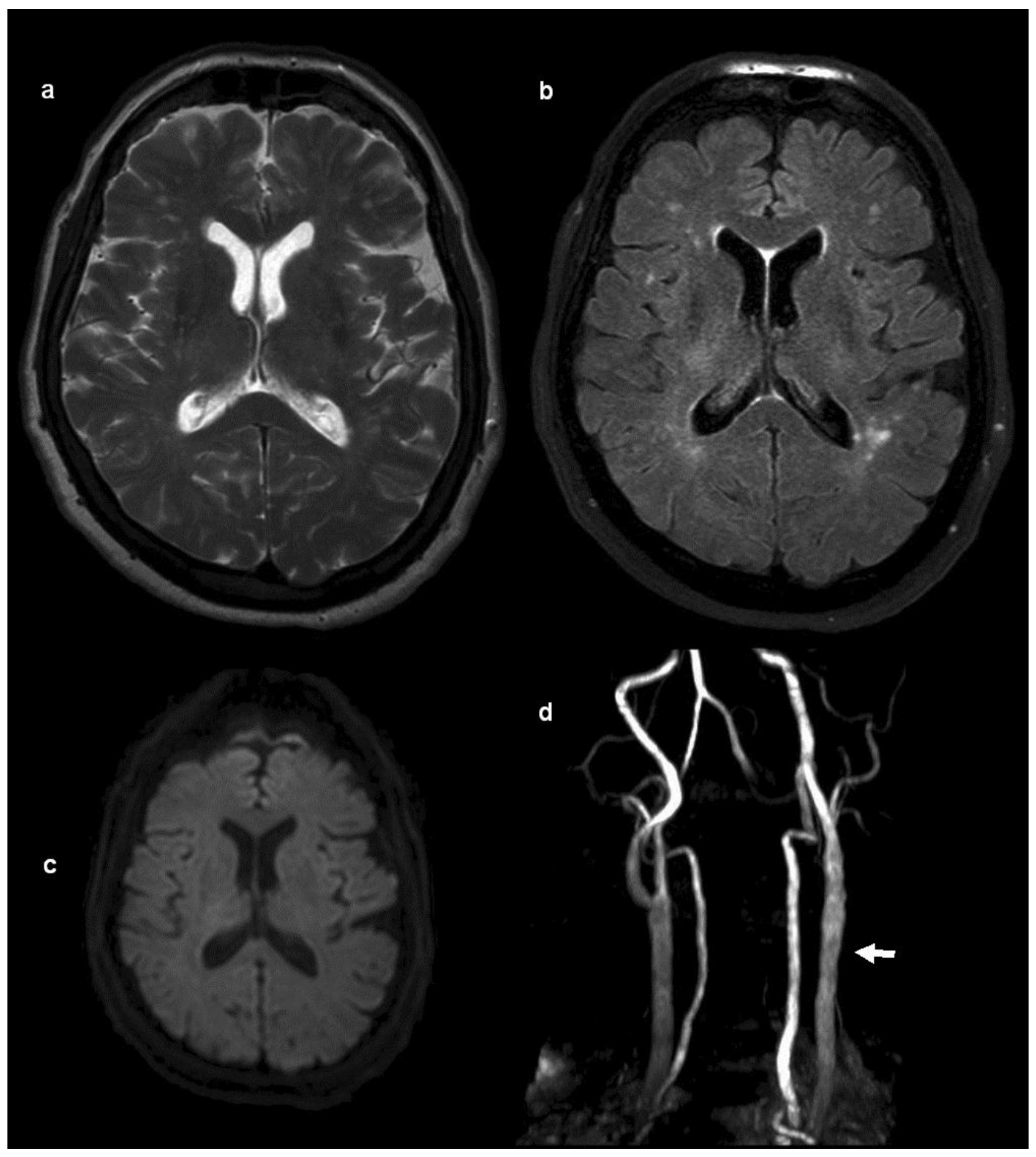

Fig. 2. At day 7 after acute right-sided hemiparesis and aphasia due to occlusion of the left carotid artery and successful surgical restoration of blood flow after $5 \mathrm{~h}$ of ischemia, T2-weighted (a), FLAIR (b), and diffusion-weighted (c) MR images of patient 1 show minor infarction changes, whereas MR angiography (d) shows now normal blood flow in the left carotid vessel (arrow). 


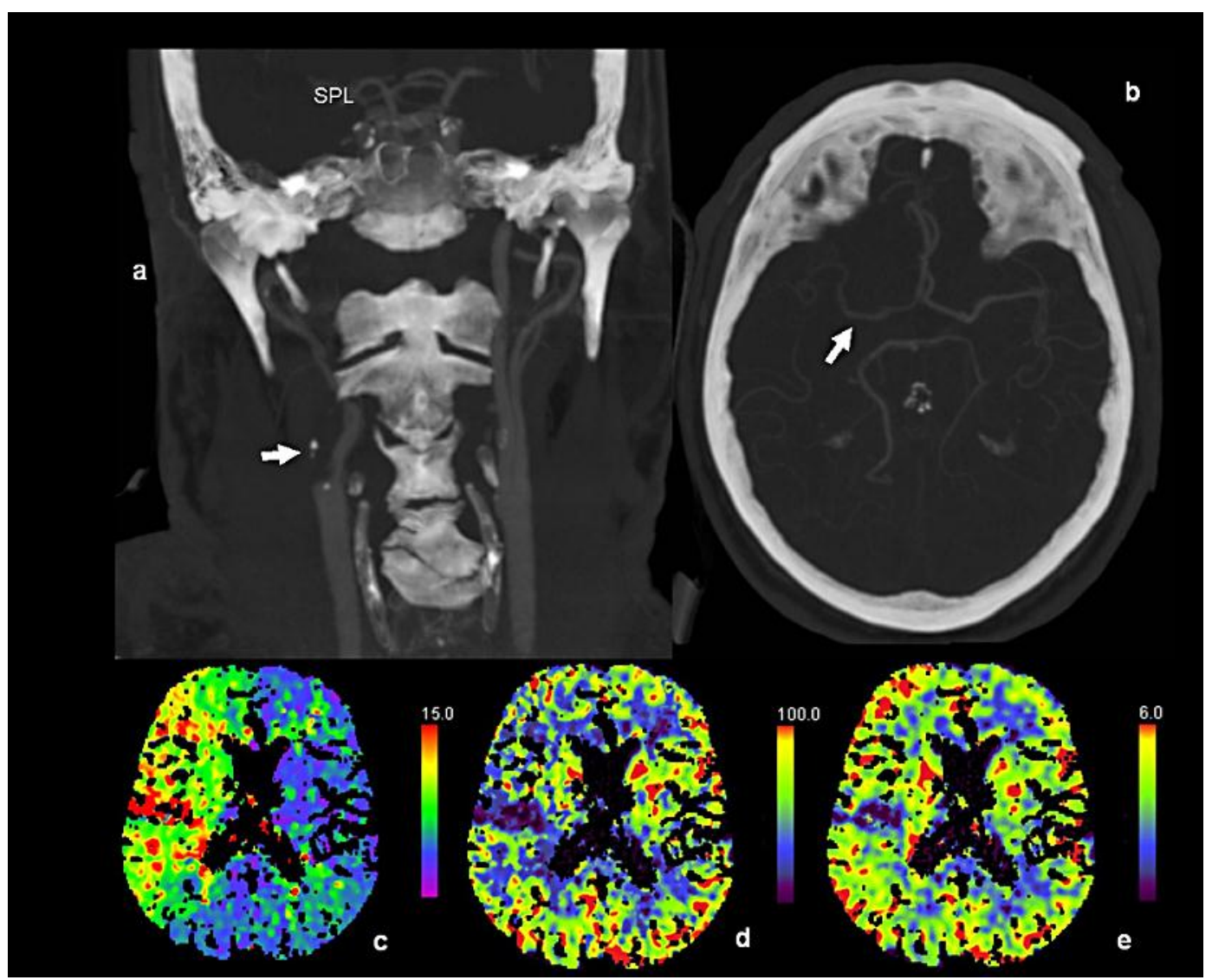

Fig. 3. Seventy minutes after onset and of left-sided hemiparesis and neglect after $8 \mathrm{~h}$ of fluctuating symptoms (patient 2). CT angiogram (a) shows total occlusion of the right ICA (arrow). Large intracranial vessels show contrast enhancement indicating collateral flow (arrow) in the right MCA (b). TTD (c), CBF (d), and CBV (e) maps show severe perfusion defect of the right hemisphere. 


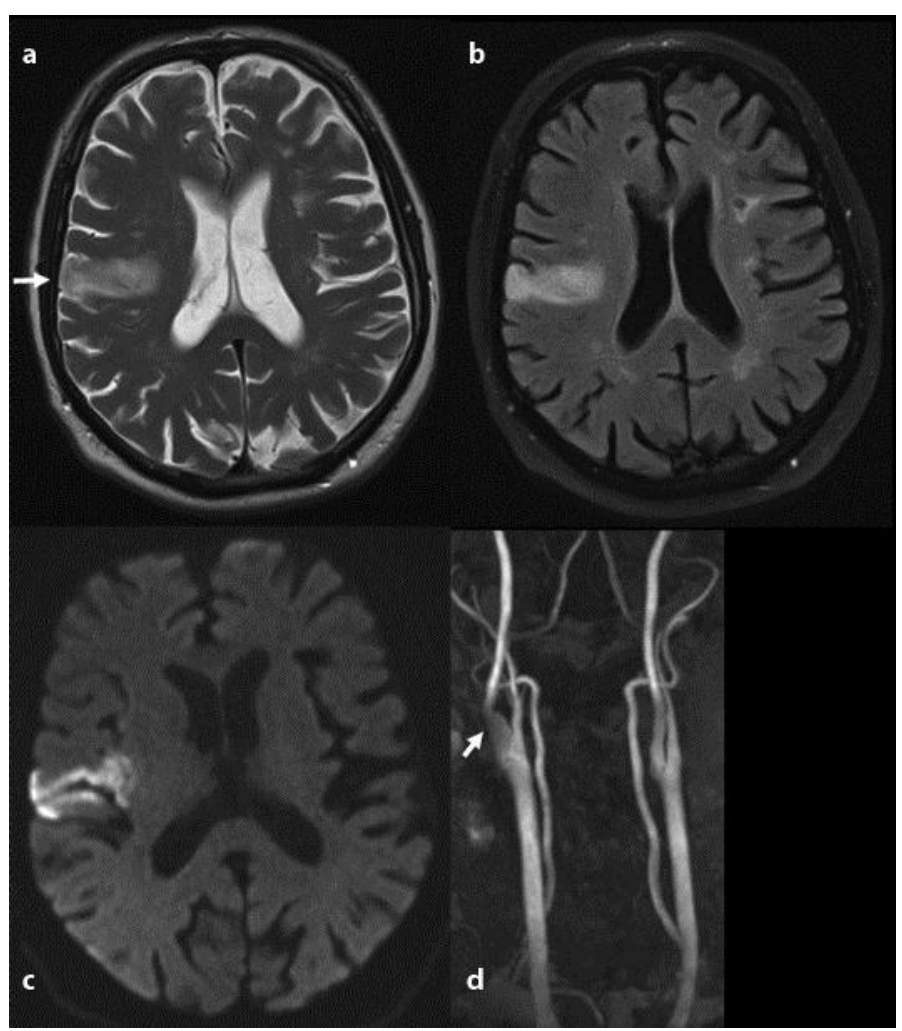

Fig. 4. At day 7 after acute left-sided hemiparesis and neglect due to occlusion of the right carotid artery and successful surgical restoration of blood flow after 5 hours of ischemia in patient 2, MR detects minor ischemic lesions on T2 (a), FLAIR (b), and DWI (c). MR angiography (d) shows now normal blood flow in the right carotid vessel (arrow).

\section{References}

1 Hacke W, Kaste M, Bluhmki E, et al: Thrombolysis with alteplase 3 to $4.5 \mathrm{~h}$ after acute ischemic stroke. N Engl J Med 2008;359:1317-1329.

2 Meyers PM, Schumacher HC, Connolly ES Jr, Heyer EJ, Gray WA, Higashida RT: Current status of endovascular stroke treatment. Circulation 2011;123:2591-2601.

-3 Meyer FB, Sundt TM Jr, Piepgras DG, Sandok BA, Forbes G: Emergency carotid endarterectomy for patients with acute carotid occlusion and profound neurological deficits. Ann Surg 1986;203:82-89.

4 Capoccia L, Sbarigia E, Speziale F, Toni D, Fiorani P: Urgent carotid endarterectomy to prevent recurrence and improve neurologic outcome in mild-to-moderate acute neurologic events. J Vasc Surg 2011;53:622-627.

5 Dorigo W, Pulli R, Nesi M, et al: Urgent carotid endarterectomy in patients with recent/crescendo transient ischaemic attacks or acute stroke. Eur J Vasc Endovasc Surg 2011;41:351-357. 